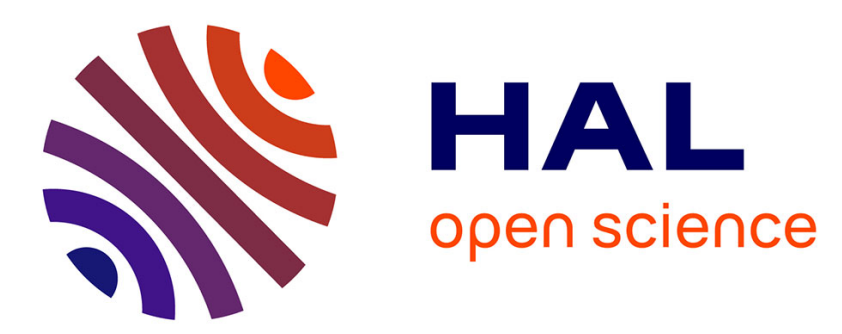

\title{
Nonlinear parabolic equation model for finite-amplitude sound propagation over porous ground layers
}

\author{
T. Leissing, P. Jean, J. Defrance, Christian Soize
}

\section{To cite this version:}

T. Leissing, P. Jean, J. Defrance, Christian Soize. Nonlinear parabolic equation model for finiteamplitude sound propagation over porous ground layers. Journal of the Acoustical Society of America, 2009, 126 (2), pp.572-581. 10.1121/1.3158937 . hal-00684342

\section{HAL Id: hal-00684342 \\ https://hal.science/hal-00684342}

Submitted on 1 Apr 2012

HAL is a multi-disciplinary open access archive for the deposit and dissemination of scientific research documents, whether they are published or not. The documents may come from teaching and research institutions in France or abroad, or from public or private research centers.
L'archive ouverte pluridisciplinaire HAL, est destinée au dépôt et à la diffusion de documents scientifiques de niveau recherche, publiés ou non, émanant des établissements d'enseignement et de recherche français ou étrangers, des laboratoires publics ou privés. 
Nonlinear parabolic equation model for finite-amplitude sound propagation over porous ground layers

\author{
Thomas Leissing, ${ }^{\text {a) }}$ Philippe Jean, and Jérôme Defrance \\ Université Paris-Est, \\ Centre Scientifique et Technique du Bâtiment (CSTB), \\ 24 rue Joseph Fourier, \\ 38400 Saint-Martin-d'Hères, \\ France \\ Christian Soize \\ Université Paris-Est, \\ Laboratoire Modélisation et Simulation Multi Echelle, \\ MSME FRE3160 CNRS, \\ 5 bd Descartes, \\ 77454 Marne-la-Vallee, \\ France
}

(Dated: March 31, 2009) 


\begin{abstract}
The nonlinear parabolic equation (NPE) is a time-domain method widely used in underwater sound propagation applications. It allows to simulate weakly nonlinear sound propagation within an inhomogeneous medium. For this method to be suited for outdoor applications, it must account for the effects of an absorbing ground surface. The NPE being formulated in the time domain, complex impedances cannot be used. The ground layer is thus included in the computational system with the help of a second NPE model based on the ZwikkerKosten model. A two-way coupling between these two layers (air and ground) is required for the whole system to behave correctly. Coupling equations are derived from linearized Euler's equations. In the frame of a parabolic model, this two-way coupling only involves spatial derivatives, making its implementation straightforward. Several propagation examples, both linear and nonlinear, are then presented and the method is shown to give satisfactory results for a wide range of ground characteristics. Finally, the problem of including Forchheimer's nonlinearities in the two-way coupling is addressed and an approximate solution is proposed.
\end{abstract}

PACS numbers: 43.25-x, 43.28.-g

Keywords: nonlinear propagation, porous ground, FDTD, parabolic 


\section{INTRODUCTION}

High-amplitudes waves propagate over large distances. The need to develop numerical models that can handle main features of finite-amplitude sound propagation outdoors is obvious. Specifically, in addition to nonlinearities, numerical models must take into account meteorological and ground effects (refraction, dissipation, hilly terrain, ground impedance).

In this work a nonlinear parabolic equation (NPE) model is used to simulate finiteamplitude sound propagation. This method has first been developed by McDonald and Kuperman in $1987^{1}$ and has been successfully used for underwater acoustics simulations ${ }^{2}$. It has also been used together with other methods to simulate blast wave propagation in $\operatorname{air}^{3-6}$.

The principle of the NPE is the resolution of a nonlinear wave equation over a moving window that surrounds the wavefront. While reducing domain size (and thus computational cost), the moving window principle prevents backward propagation to be accounted for. For the derivation of the original NPE model, the reader may refer to articles by McDonald ${ }^{7,8}$ or Caine and West ${ }^{9}$. The NPE model for a 2D domain with cartesian coordinates $(x, z)$ writes:

$$
D_{t} R=-\partial_{x}\left(c_{1} R+c_{0} \frac{\beta}{2} R^{2}\right)-\frac{c_{0}}{2} \int \partial_{z}^{2} R d x
$$

where $\partial_{i}$ means partial derivation with respect to the variable $i, x$ is the main propagation direction, $z$ is the transverse propagation direction and $t$ is the time variable. The ambient sound speed is $c_{0}$ while $c_{1}$ is the sound speed perturbation in the window, i.e. $c_{1}=c(x, z)-$ $c_{0}$, where $c(x, z)$ is the spatially-dependent sound speed. $R=\rho^{\prime} / \rho_{0}$ is a dimensionless overdensity variable, with $\rho^{\prime}$ the acoustic density perturbation and $\rho_{0}$ the ambient medium density. For air, the coefficient of nonlinearity $\beta$ is calculated with the help of the ratio of specific heat capacities at constant volume and pressure $\gamma$, i.e. $\beta=(\gamma+1) / 2$. The first term on the right hand side of Eq. (1) simulates refraction and nonlinear effects; the second term accounts for propagation in the transverse direction. $D_{t}$ is a moving window operator

\footnotetext{
${ }^{a)}$ Corresponding author; Electronic address: thomas.leissing@cstb.fr
} 
and is defined by:

$$
D_{t}=\partial_{t}+c_{0} \partial_{x}
$$

Note that in Eq. (1), the azimuthal spreading term $c_{0} R /(2 r)$ has been dropped from the original $\mathrm{NPE}^{1}$. The assumptions used to derive this model are: $(i)$ weak nonlinearities; $(i i)$ weak sound speed perturbations, i.e. $c_{1} \ll c_{0}$; (iii) propagation along a main direction. Eq. (1) can thus be used to propagate weak shocks over moderate distances within a domain with spatially-varying sound speed. Various modifications and additions to this original model were made during the past two decades: spherical and cylindrical formulations ${ }^{10}$, thermoviscous effects $^{11}$, high-angle formulation ${ }^{12}$. Propagation in multiple media ${ }^{13}$ and propagation through atmospheric turbulences ${ }^{14}$ were successfully studied using this model.

Euler's equations methods can provide complete solutions to nonlinear sound propagation problems ${ }^{15}$ : realistic absorption models ${ }^{16}$, meteorological effects ${ }^{17,18}$, hilly terrain ${ }^{19}$ and ground impedances ${ }^{20-23}$ can be accounted for in a very accurate way. Moreover, it does not suffer from the parabolic approximation inherent to NPE models. However, for long-range wave propagation problems, computational times will often be on the order of days for 3D domains. Despite increasing computational resources and the existence of modern numerical techniques, such as the use of efficient absorbing layers ${ }^{24}$ or adaptive mesh refinement methods $^{25}$, Euler's equations-based models cannot vie in calculation time with NPE-based methods. Indeed, the use of a one-variable wave equation makes the NPE an efficient tool for studying long-range sound propagation. The main motivation for the development of the NPE model presented here is its use to study finite-amplitude wave propagation over urban environments. Reduced computational times will allow this model to be used several hundred times to obtain statistical information on the wave fields.

In the present work, a parabolic model that takes into account the effects of a soft ground layer on propagation is proposed. It is not the objective here to study the propagation within the ground layer but rather to capture the effects of the non-rigid interface on the air acoustic fields. The derivation of the NPE model for porous ground layers is described in section II. Combined with two-way coupling equations, presented in section III, and a NPE model 
for atmospheric media, it allows to simulate finite-amplitude sound propagation over an impedant ground surface. Several propagation examples are then shown and finally, an approximate solution to include Forchheimer's nonlinearities ${ }^{26,27}$ in the two-way coupling is presented.

\section{NPE MODEL FOR RIGIDLY-FRAMED POROUS MEDIA}

The domain considered is two-dimensional with main axes $x$ (horizontal direction) and $z$ (vertical direction). Total density $\rho_{T}$ and total pressure $p_{T}$ variables are noted as follows:

$$
\rho_{T}=\rho_{0}+\rho^{\prime} \quad p_{T}=p_{0}+p^{\prime}
$$

where $\rho_{0}$ and $p_{0}$ are ambient air density and ambient air pressure, respectively, and $\rho^{\prime}$ and $p^{\prime}$ are acoustic perturbations of these quantities. Components of the flow velocity vector $\mathbf{V}$ are $u$ and $w$, which are the flow velocities in the $x$ - and $z$-directions, respectively.

It is reminded that the effects of a soft ground on sound propagation in the air layer is under interest. Moreover, including the porous medium into the computational system must not dramatically increase computational times, otherwise one of the most interesting feature of NPE models, fast calculations, will be lost. It is thus proposed to derive a parabolic model similar to Eq. (1), which uses a minimal parameterization: the layer is assumed to be equivalent to a continuous fluid for sound waves. A wave causes a vibration of air particles contained in the ground pores, while the ground frame does not vibrate. The nonlinear parabolic equation model for sound propagation in porous ground media is based on a nonlinear extension of the Zwikker-Kosten (ZK) model $^{28}$, characterized by a set of 4 parameters: the DC flow resistivity $\sigma_{0}$, the porosity $\Omega_{0}$, the tortuosity $\Phi$ and the Forchheimer's nonlinearity parameter $\xi$. The tortuosity $\Phi$ is defined as the ratio of a curved path length to the distance between its end points. These quantities are assumed fixed in

space and time. In this context, equations of continuity and conservation of momentum 
$\operatorname{are}^{29-31}$ :

$$
\begin{gathered}
\partial_{t} \rho_{T}+\partial_{x}\left(\rho_{T} u\right)+\partial_{z}\left(\rho_{T} w\right)=0 \\
\Phi \partial_{t}\left(\rho_{T} u\right)+\partial_{x}\left(p_{T}+\Phi \rho_{T} u^{2}\right)+\partial_{z}\left(\Phi \rho_{T} u w\right) \\
+\sigma_{0} \Omega_{0}(1+\xi|u|) u=0 \\
\Phi \partial_{t}\left(\rho_{T} w\right)+\partial_{z}\left(p_{T}+\Phi \rho_{T} w^{2}\right)+\partial_{x}\left(\Phi \rho_{T} u w\right) \\
+\sigma_{0} \Omega_{0}(1+\xi|w|) w=0
\end{gathered}
$$

As one can see in Eqs. (4), the tortuosity $\Phi$ reduces the pressure gradients and flow resistive terms. Combining Eqs. (4) gives:

$$
\begin{aligned}
& \Phi \partial_{t}^{2} \rho_{T}=\partial_{x}^{2}\left(p_{T}+\Phi \rho_{T} u^{2}\right) \\
& +\partial_{z}^{2}\left(p_{T}+\Phi \rho_{T} w^{2}\right)+2 \partial_{x} \partial_{z}\left(\Phi \rho_{T} u w\right) \\
& +\sigma_{0} \Omega_{0} \partial_{x}[(1+\xi|u|) u]+\sigma_{0} \Omega_{0} \partial_{z}[(1+\xi|w|) w]
\end{aligned}
$$

Since the propagation is mainly along the $x$-direction, only linear terms in $z$-derivatives are kept in Eq. (5): terms $\partial_{x} \partial_{z}\left(\Phi \rho_{T} u w\right), \partial_{z}^{2}\left(\Phi \rho_{T} w^{2}\right)$ and $\sigma_{0} \Omega_{0} \partial_{z}(\xi|w| w)$ are neglected. Moreover, only terms of order up to two in $x$-derivatives are retained: the quantity $\partial_{x}^{2}\left(\Phi \rho^{\prime} u^{2}\right)$ is discarded; this leads to:

$$
\begin{aligned}
\Phi \partial_{t}^{2} \rho_{T} & =\partial_{x}^{2}\left(p_{T}+\Phi \rho_{0} u^{2}\right)+\partial_{z}^{2} p_{T} \\
& +\sigma_{0} \Omega_{0} \partial_{x}[(1+\xi|u|) u]+\sigma_{0} \Omega_{0} \partial_{z} w
\end{aligned}
$$

To find an expression for the flow velocities $u$ and $w$ we use the perturbation expansions method. The same scalings and expansions as in references ${ }^{1,7}$ are used (however, note that the window speed in the ground layer is set to $\left.c_{0} / \sqrt{\Phi}\right)$ :

$$
x \longrightarrow x-\frac{c_{0}}{\sqrt{\Phi}} t \quad z \longrightarrow \epsilon^{1 / 2} z \quad t \longrightarrow \epsilon t
$$

The scaling of $z$ by a factor of $\epsilon^{1 / 2}$ emphasizes the predominance of the propagation in the $x$-direction. The partial derivatives associated with Eqs. (7) are:

$$
\partial_{x} \longrightarrow \partial_{x} \quad \partial_{z} \longrightarrow \epsilon^{1 / 2} \partial_{z} \quad \partial_{t} \longrightarrow \epsilon \partial_{t}-\frac{c_{0}}{\sqrt{\Phi}} \partial_{x}
$$


The dependent variables are expanded as follows:

$$
\begin{gathered}
\rho_{T} \longrightarrow \rho_{0}+\epsilon \rho_{1}+\epsilon^{2} \rho_{2}+\epsilon^{3} \rho_{3}+\cdots \\
u \longrightarrow \epsilon u_{1}+\epsilon^{3 / 2} u_{2}+\epsilon^{2} u_{3}+\cdots \\
w \longrightarrow \epsilon w_{1}+\epsilon^{3 / 2} w_{2}+\epsilon^{2} w_{3}+\cdots
\end{gathered}
$$

Eq. (4a) can then be rewritten:

$$
\begin{gathered}
\left(\epsilon \partial_{t}-\frac{c_{0}}{\sqrt{\Phi}} \partial_{x}\right)\left(\rho_{0}+\epsilon \rho_{1}^{\prime}+\epsilon^{2} \rho_{2}^{\prime}+\cdots\right)=-\partial_{x}\left[\left(\rho_{0}+\epsilon \rho_{1}+\epsilon^{2} \rho_{2}+\cdots\right)\left(\epsilon u_{1}+\epsilon^{3 / 2} u_{2}+\cdots\right)\right] \\
-\epsilon^{1 / 2} \partial_{z}\left[\left(\rho_{0}+\epsilon \rho_{1}+\epsilon^{2} \rho_{2}+\cdots\right)\left(\epsilon w_{1}+\epsilon^{3 / 2} w_{2}+\cdots\right)\right]
\end{gathered}
$$

Equating terms of order $\epsilon$ and $\epsilon^{3 / 2}$ gives:

$$
u_{1}=\frac{c_{0}}{\sqrt{\Phi}} \frac{\rho_{1}}{\rho_{0}} \quad w_{1}=0
$$

Note that $\rho^{\prime}=\rho_{1}+O\left(\epsilon^{2}\right), u=u_{1}+O\left(\epsilon^{3 / 2}\right)$ and $w=w_{1}+O\left(\epsilon^{3 / 2}\right)$. Substitution of $u$ and $w$ by $u_{1}$ and $w_{1}$ in Eq. (6) leads to an error consistent with the accuracy sought.

The total pressure $p_{T}$ is then substituted by a second-order expansion in $\rho^{\prime}$ from an assumed adiabatic equation of state:

$$
p_{T}=p_{0}+c_{0}^{2} \rho^{\prime}+c_{0}^{2}\left(\frac{\gamma-1}{2 \rho_{0}}\right) \rho^{\prime 2}
$$

where $\gamma$ is the ratio of specific heats. Inserting Eq. (12) in Eq. (6) yields:

$$
\begin{aligned}
\Phi \partial_{t}^{2} \rho^{\prime} & =c_{0}^{2} \partial_{x}^{2}\left[\rho^{\prime}+\left(\frac{\gamma+1}{2 \rho_{0}}\right) \rho^{2}\right]+c_{0}^{2} \partial_{z}^{2} \rho^{\prime} \\
& +\frac{\sigma_{0} \Omega_{0} c_{0}}{\rho_{0} \sqrt{\Phi}} \partial_{x}\left[\left(1+\frac{\xi c_{0}}{\sqrt{\Phi}}\left|\frac{\rho^{\prime}}{\rho_{0}}\right|\right) \rho^{\prime}\right]
\end{aligned}
$$

A moving-frame operator $D_{t}^{\star}$ is introduced:

$$
D_{t}^{\star}=\partial_{t}+\frac{c_{0}}{\sqrt{\Phi}} \partial_{x}
$$

The first-order parabolic approximation gives ${ }^{9}$ :

$$
\partial_{t}^{2} \longrightarrow-2 \frac{c_{0}}{\sqrt{\Phi}} D_{t}^{\star} \partial_{x}+\frac{c_{0}^{2}}{\Phi} \partial_{x}^{2}
$$


Replacing the second time derivative in Eq. (13) and rearranging gives a NPE model for propagation in porous media:

$$
\begin{aligned}
D_{t}^{\star} R= & -\frac{c_{0}}{\sqrt{\Phi}} \partial_{x}\left(\frac{\beta}{2} R^{2}\right)-\frac{c_{0}}{2 \sqrt{\Phi}} \int \partial_{z}^{2} R d x \\
& -\frac{\sigma \Omega}{2 \Phi \rho_{0}}\left(1+\frac{\xi c_{0}}{\sqrt{\Phi}}|R|\right) R
\end{aligned}
$$

Eq. (16) can be used to simulate sound propagation within a porous ground layer. However, if one wants to couple air/ground models, a last modification must be done. Indeed, both models use different moving-window speeds: $c_{0}$ and $c_{0} / \sqrt{\Phi}$. Correcting for the frame-speed difference leads to the following substitution:

$$
D_{t}^{\star} \longrightarrow D_{t}+\frac{c_{0}}{\sqrt{\Phi}}(1-\sqrt{\Phi}) \partial_{x}
$$

Eq. (16) becomes:

$$
D_{t} R=-\frac{c_{0}}{\sqrt{\Phi}} \partial_{x}\left[(1-\sqrt{\Phi}) R+\frac{\beta}{2} R^{2}\right]-\frac{c_{0}}{2 \sqrt{\Phi}} \int \partial_{z}^{2} R d x-\frac{\sigma_{0} \Omega_{0}}{2 \Phi \rho_{0}}\left(1+\frac{\xi c_{0}}{\sqrt{\Phi}}|R|\right) R
$$

The NPE model described by Eq. (18) is able to simulate finite amplitude sound propagation within a rigidly-framed porous material described by a set of 4 parameters. Note that if one sets $\Phi=1$ and neglects losses in the layer, i.e. $\sigma_{0}=0$, the model exactly reduces to the usual NPE model for atmospheric propagation Eq. (1). Looking at Eq. (18) allows to draw some conclusions about finite-amplitude sound propagation in porous media: $(i)$ the sound speed in the medium is inversely proportional to the square root of the material tortuosity, i.e. $c=c_{0} / \sqrt{\Phi} ;(i i)$ the attenuation in the ground layer is composed of a linear term plus a nonlinear term; (iii) in the frame of this model, the material resistivity is proportional to the overdensity $R$.

\section{DERIVATION OF TWO-WAY COUPLING EQUATIONS}

As both models use the same moving-frame speed, they can be combined to simulate finite-amplitude sound propagation over a rigidly-framed porous ground layer. This section 
aims at establishing first-order coupling equations to link these two propagation models. In the following we assume that the deformation of the interface by the wave is small ${ }^{13}$.

\section{A. Derivation}

An air layer, which fields are noted $p^{a}, u^{a}$ and $w^{a}$, is considered. To construct the air-ground interfacial condition, a rigidly-framed porous ground layer is introduced. Its fields are noted $p^{g}, u^{g}$ and $w^{g}$. With these notations, interfacial boundary conditions are continuity of pressure and normal flow velocity:

$$
\left[p^{\prime a}\right]=\left[p^{g}\right] \quad\left[w^{a}\right]=\left[w^{g}\right]
$$

where the square brackets denote the field quantity on the air-ground interface. Expressions of $w^{a}$ and $w^{g}$ involving the pressure disturbance $p^{\prime}$ to the first order are sought. As a first order boundary interface condition is sought, linearized equations are used; for the air layer we use the linearized Euler's equation:

$$
\rho_{0} \partial_{t}\left(w^{a}\right)=-\partial_{z} p_{T}^{a}
$$

The perturbation expansion method is used and the same scalings as in section II and in references $^{1,7}$ are used. Rewriting Eq. (20) and equating terms of order 1 and 3/2 gives:

$$
w_{1}^{a}=0 \quad w_{2}^{a}=\left(\rho_{0} c_{0} \partial_{x}\right)^{-1} \partial_{z} p_{1}^{\prime a}
$$

Note that $w^{a}=w_{1}^{a}+w_{2}^{a}+O\left(\epsilon^{5 / 2}\right)$. To the order of accuracy sought in this work it can be written:

$$
w^{a}=\left(\rho_{0} c_{0} \partial_{x}\right)^{-1} \partial_{z} p_{1}^{a}
$$

To find an expression for $w^{g}$ we start from the following equation ${ }^{20}$ :

$$
\Phi \rho_{0} \partial_{t} w^{g}=-\Omega_{0} \partial_{z} p_{T}^{g}-\sigma_{0} \Omega_{0} w^{g}
$$

The same procedure is applied; one can find:

$$
w^{g}=\left(\sqrt{\Phi} \rho_{0} c_{0} \partial_{x}-\sigma_{0} \Omega_{0}\right)^{-1} \Omega_{0} \partial_{z} p_{1}^{g}
$$


The interfacial condition for the continuity of vertical velocities $w^{a}$ and $w^{g}$ can now be written:

$$
\left[\left(\rho_{0} c_{0} \partial_{x}\right)^{-1} \partial_{z} p^{\prime a}\right]=\left[\left(\sqrt{\Phi} \rho_{0} c_{0} \partial_{x}-\sigma_{0} \Omega_{0}\right)^{-1} \Omega_{0} \partial_{z} p^{\prime g}\right]
$$

Rearranging Eq. (25) leads to:

$$
\left[\sqrt{\Phi} \partial_{z} p^{\prime a}-\frac{\sigma_{0} \Omega_{0}}{\rho_{0} c_{0}} \int \partial_{z} p^{\prime a} d x\right]=\left[\Omega_{0} \partial_{z} p^{\prime g}\right]
$$

\section{B. Discretization}

The variables $p_{i, j}^{\prime a}$ and $p_{i, j}^{\prime g}$ are introduced to denote pressure values in layer $a$ (air layer) and layer $g$ (porous ground layer), respectively, at range $i \Delta x$ in the moving window and altitude $j \Delta z$. The air-ground interface is taken to be midway between two vertical grid points with indexes $j=0$ and $j=1$. Auxiliary virtual points with pressure values $p_{i, 0}^{\prime a}$ and $p_{i, 1}^{\prime g}$ are created. Fig. 1 shows a sketch of the configuration.

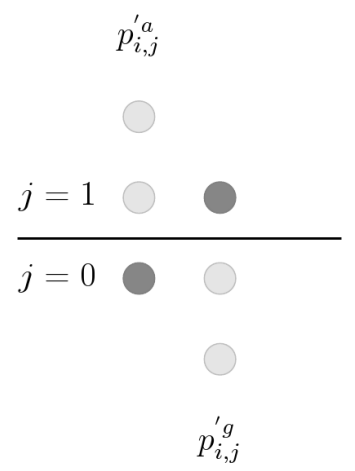

FIG. 1. The air-ground interface is taken to be midway between two vertical grid points with indexes $j=0$ and $j=1$. Auxiliary virtual points (black circles) with pressure values $p_{i, 0}^{\prime a}$ and $p_{i, 1}^{\prime g}$ are created.

A trapezoïdal law and finite-differences expressions for $p^{\prime a}$ and $p^{\prime g}$ and their derivatives are used to discretize Eq. (26). For a layer $l$ we use:

$$
\begin{aligned}
{\left[p^{\prime} l\right] } & =\frac{p_{i, 1}^{\prime l}+p_{i, 0}^{\prime l}}{2} \\
{\left[\partial_{z} p^{\prime l}\right] } & =\left(p_{i, 1}^{\prime l}-p_{i, 0}^{\prime l}\right) \Delta z^{-1}
\end{aligned}
$$


Replacing these approximations into Eq. (26) and using the condition of pressure equality across the interface finally gives expressions for unknown quantities $p_{i, 0}^{\prime a}$ and $p_{i, 1}^{\prime g}$ :

$$
\begin{aligned}
& (A+G) p_{i, 0}^{\prime a}=(A-G) p_{i, 1}^{\prime a}+2 G p_{i, 0}^{\prime g}+S \sum_{m=N_{x}}^{i+1}\left(p_{m, 1}^{\prime a}-p_{m, 0}^{\prime a}\right) \\
& (A+G) p_{i, 1}^{\prime g}=(G-A) p_{i, 0}^{\prime g}+2 A p_{i, 1}^{\prime a}+S \sum_{m=N_{x}}^{i+1}\left(p_{m, 1}^{\prime a}-p_{m, 0}^{\prime a}\right)
\end{aligned}
$$

where $N_{x}$ is the number of points in the moving window in the $x$-direction and:

$$
\begin{aligned}
& A=\sqrt{\Phi}+\frac{1}{2} S \\
& G=\Omega_{0} \\
& S=\frac{\sigma_{0} \Omega_{0} \Delta x}{c_{0} \rho_{0}}
\end{aligned}
$$

Eqs. (28) and (29) give expressions for the unknown pressures $p_{i, 0}^{a}$ and $p_{i, 1}^{g}$, and thus allow, used together with the atmospheric and porous ground NPE models, to simulate weakly nonlinear sound propagation over an impedant ground.

\section{Properties}

In this section, fundamental properties of the boundary conditions are described and some notes about its numerical implementation are given.

Limitations: first-order formulations of the constitutive equations have been used to derive the boundary interface condition. This implies that nonlinearities can't be taken into account in the two-way coupling.

Causality: the $x$-integral present in NPE models (see for example Eq. (1)) is calculated from the right to the left of the calculation grid, and the same method is used for coupling (note the reversed sum indexes in Eqs. (28)). This ensures that no perturbation is introduced ahead of the point where the wave hits the ground, and thus implies that the interfacial condition is causal. 
Consistency to classical boundary conditions: if one sets $\Phi=+\infty$ we obtain from Eqs. (28): $p_{i, 0}^{\prime a}=p_{i, 1}^{\prime a}$ which is, with the discretization used, the condition for a totally rigid interface. A transparent interface condition can be obtained by setting $\sigma_{0}=0, \Omega_{0}=1$ and $\Phi=1$ (parameters for an air layer). This leads to: $A=1$ and $G=1$ and thus $p_{i, 0}^{\prime a}=p_{i, 0}^{\prime g}$ and $p_{i, 1}^{\prime g}=p_{i, 1}^{\prime a}$ which is the condition for perfect transmission. If one sets $\sigma=0$ and $\Omega_{0}=1$, Eqs. (28) become:

$$
\begin{aligned}
p_{i, 0}^{\prime a} & =\frac{\sqrt{\Phi}-1}{\sqrt{\Phi}+1} p_{i, 1}^{\prime a}+\frac{2}{\sqrt{\Phi}+1} p_{i, 0}^{\prime g} \\
p_{i, 1}^{\prime g} & =\frac{1-\sqrt{\Phi}}{\sqrt{\Phi}+1} p_{i, 0}^{\prime g}+\frac{2 \sqrt{\Phi}}{\sqrt{\Phi}+1} p_{i, 1}^{\prime a}
\end{aligned}
$$

which is the interface condition for two fluid layers with densities $\rho_{0}$ and $\sqrt{\Phi} \rho_{0}{ }^{13}$.

Numerical implementation: a common way for solving for the diffraction operator is to use first-order finite-differences approximation for spatial discretization and Crank-Nicolson method for time marching. This leads to a tridiagonal system of equations that is solved columnwise, from the right to the left of the calculation grid. The boundary interface condition can thus be naturally included in the diffraction solver by imposing values on corresponding points without any additional solver modifications.

\section{NUMERICAL EXAMPLES}

In this section numerical examples of sound propagation over porous ground layers are presented to illustrate the coupling method and evaluate its performances.

\section{A. Linear propagation}

\section{Reference solutions}

Solutions of the two-dimensional Helmoltz equation are used as references. The solution for the propagation in an homogeneous atmosphere over an impedant ground surface is (for 
2-dimensional waves):

$$
p_{r}=i \pi H_{0}^{(1)}\left(k R_{1}\right)+Q i \pi H_{0}^{(1)}\left(k R_{2}\right)
$$

where $p_{r}$ is the complex pressure at the receiver, $k$ is the wavenumber, $R_{1}$ and $R_{2}$ are the

source-receiver and image source-receiver distances, respectively, and $H_{0}^{(1)}$ is the Hankel function of the first kind and order zero. $Q$ is the cylindrical reflection coefficient and can be calculated with the help of Laplace transforms ${ }^{20,32}$. The normalized impedance used to calculate the reflection coefficient is ${ }^{20}$ :

$$
Z=\sqrt{\frac{\Phi}{\Omega_{0}^{2}}+i \frac{\sigma_{0}}{\rho_{0} \Omega_{0} \omega}}
$$

\section{Configuration}

The sound speed is constant within the domain $\left(c_{0}=340 \mathrm{~m} . \mathrm{s}^{-1}\right)$, and there is no absorption from air included. Waves decay at a cylindrical rate. The source is positioned at $\left(x_{s}, z_{s}\right)=(0.0,1.4) m$ and the signal used is a sine pulse with wavelength $\lambda=0.27$ $\mathrm{m}(f=1259.25 \mathrm{~Hz})$ and peak amplitude low enough for the propagation to be considered linear. A virtual receiver is placed $10 \mathrm{~m}$ away from the source and at altitude $z=1.4 \mathrm{~m}$. The receiver position ensures that we are within the parabolic equation angular validity domain (the angle from source to image-receiver is $\theta \approx 15^{\circ}$ ). Spatial steps are equal to $7.510^{-3} \mathrm{~m}$ in both directions, thus giving a spatial resolution of about 36 points $/ \lambda$, ensuring sufficient resolution at higher frequencies and near the air/ground boundary. The time step is $\Delta x / c_{0}$, so that for each time step the window advances one spatial step. Since semi-implicit schemes are used (Crank-Nicolson method) the numerical scheme is stable. Three different ground layers of thickness 1 meter are considered. The first ground layer is a perfectly rigid surface $(\Phi \gg 1)$. The second and third layers have identical tortuosity $(\Phi=3)$ and porosity $\left(\Omega_{0}=0.3\right)$, but different flow resistivities $\left(\sigma_{0}=500 \mathrm{kPa} . \mathrm{s} . \mathrm{m}^{-2}\right.$ and $\left.\sigma_{0}=100 \mathrm{kPa} . \mathrm{s} . \mathrm{m}^{-2}\right)$. The NPE window including the ground layer is 3 meters wide and 4.125 meters high (400 by 550 points). 


\section{Results}

Time signals are recorded at the virtual receiver $\left(x_{r}=10 \mathrm{~m}, z_{r}=1.4 \mathrm{~m}\right)$; two modifications are done on raw signals: first, in order to obtain a free field reference, time histories are cropped after the direct wave. Low-amplitude numerical oscillations appear on the trailing part of time signals: for propagation on the perfectly rigid layer, these oscillations prevents obtaining complete destructive interferences $(\Delta L \mapsto-\infty)$; cropping original signals after the reflected wave allows to eliminate this unwanted numerical noise. Relative sound pressure levels (SPLs) $\Delta L$ are then calculated with:

$$
\Delta L=10 \log \left(\frac{p_{r}^{2}}{p_{\text {free }}^{2}}\right)
$$

where $p_{r}$ and $p_{\text {free }}$ are the Fourier-transformed receiver and free field signals. Note that $\mathrm{in}^{19}$, it has been found that the difference on relative SPLs is very small when comparing sources with different decay rates. Analytical solutions for cylindrical line sources are thus valid references for comparison with the NPE model used in this work.

Relative SPLs at the receiver are shown in Fig. 2, for both analytical and NPE calculations. Very good agreement can be observed, independantly of the ground properties: even for the softest layer $\left(\sigma_{0}=100 \mathrm{kPa} . \mathrm{s} . \mathrm{m}^{-2}\right)$ the difference between analytical and NPE calculations is at most $1 \mathrm{~dB}$. The frequencies where negative interference occurs are 1325 $\mathrm{Hz}, 1273 \mathrm{~Hz}$ and $1246 \mathrm{~Hz}$ for the rigid case, ground layer with $\sigma_{0}=500 \mathrm{kPa} . \mathrm{s} . \mathrm{m}^{-2}$ and ground layer with $\sigma_{0}=100 \mathrm{kPa} . \mathrm{s} . \mathrm{m}^{-2}$, respectively. As one can see on Fig. 2 the NPE model presented does not only accurately recreate reflected wave amplitude decrease, but does account for the change of least reflective frequencies due to the additional delay given during reflection. 


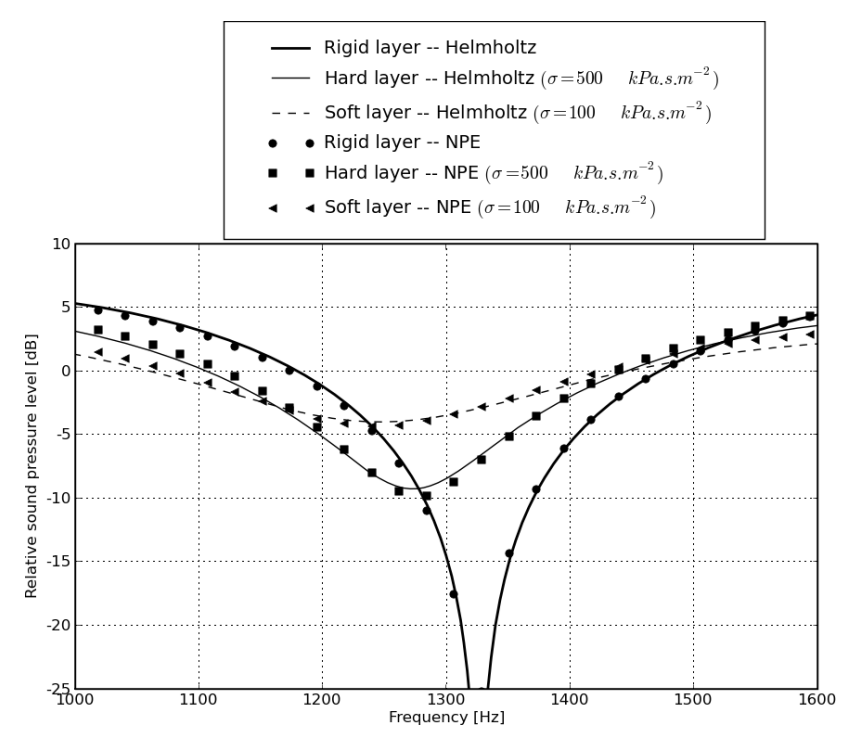

FIG. 2. SPLs relative to free field at the receiver for the three different ground layers, for NPE and analytical solutions. The source and receiver are placed at altitude $z_{s}=1.4 \mathrm{~m}$; the receiver is $10 \mathrm{~m}$ away from the source.

\section{B. Nonlinear propagation}

\section{Reference solution}

To obtain reference results to compare to the NPE model, solutions of the Euler's equations are used. The computational domain is composed of an air and a ground layer. In a two-dimensional Cartesian coordinate system the constitutive equations for the air layer are:

$$
\begin{gathered}
\partial_{t} \rho_{T}+\partial_{x}\left(\rho_{T} u\right)+\partial_{z}\left(\rho_{T} w\right)=0 \\
\partial_{t}\left(\rho_{T} u\right)+\partial_{x}\left(\rho_{T} u^{2}\right)+\partial_{z}\left(\rho_{T} u w\right)=-\partial_{x} p_{T} \\
\partial_{t}\left(\rho_{T} w\right)+\partial_{x}\left(\rho_{T} u w\right)+\partial_{z}\left(\rho_{T} w^{2}\right)=-\partial_{z} p_{T} \\
\partial_{t}\left(\rho_{T} e_{0}\right)+\partial_{x}\left(\rho_{T} u e_{0}\right)+\partial_{z}\left(\rho_{T} w e_{0}\right)= \\
-\partial_{x}\left(p_{T} u\right)-\partial_{z}\left(p_{T} w\right)
\end{gathered}
$$


where $e_{0}$ is the energy per unit mass. Within the ground layer momentum conservation equations write:

$$
\begin{gathered}
\Phi \partial_{t}\left(\rho_{T} u\right)+\partial_{x}\left(p_{T}+\Phi \rho_{T} u^{2}\right)+\partial_{z}\left(\Phi \rho_{T} u w\right) \\
+\sigma_{0} \Omega_{0}(1+\xi|u|) u=0 \\
\Phi \partial_{t}\left(\rho_{T} w\right)+\partial_{z}\left(p_{T}+\Phi \rho_{T} w^{2}\right)+\partial_{x}\left(\Phi \rho_{T} u w\right) \\
+\sigma_{0} \Omega_{0}(1+\xi|w|) w=0
\end{gathered}
$$

The energy equation Eq. (36) and the ideal gas law Eq. (37) close the equation system:

$$
\begin{gathered}
\rho_{T} e_{0}=\rho_{T} C_{v} T+\frac{\rho_{T}|\mathbf{V}|^{2}}{2} \\
p_{T}=\rho_{T} R T
\end{gathered}
$$

where $T$ is the gas temperature, $C_{v}$ is the specific heat capacity at constant volume and $R$ is the gas constant. To solve this equation system a weighted essentially non-oscillatory (WENO) algorithm ${ }^{33}$ for space discretization and a third-order total variation diminishing (TVD) scheme ${ }^{34}$ for time marching are used. These numerical algorithms are briefly presented in Appendix A.

\section{Configuration}

In this example standard atmospheric conditions are used $\left(T=293 \mathrm{~K}, \rho_{0}=1.2 \mathrm{~kg} \cdot \mathrm{m}^{-3}\right.$, $p_{0}=1.0310^{5} \mathrm{~Pa}$ ). The source is positioned $3 \mathrm{~m}$ high; the receiver is $12 \mathrm{~m}$ away from the source at the same altitude.

In order to start the reference calculation, the pressure, velocity, density and energy need to be specified. A Gaussian pulse is propagated using a one-dimensional version of the code presented in Appendix A. By adjusting the pulse amplitude and width, one can obtain a one dimensional signal at a given distance. In this example, an amplitude and signal length of approximately $4 \mathrm{kPa}$ and $1.5 \mathrm{~m}$, respectively, were aimed for at a distance of $3 \mathrm{~m}$ from the source. Spatial steps are equal to $0.015 \mathrm{~m}$ in both directions, leading to a resolution 

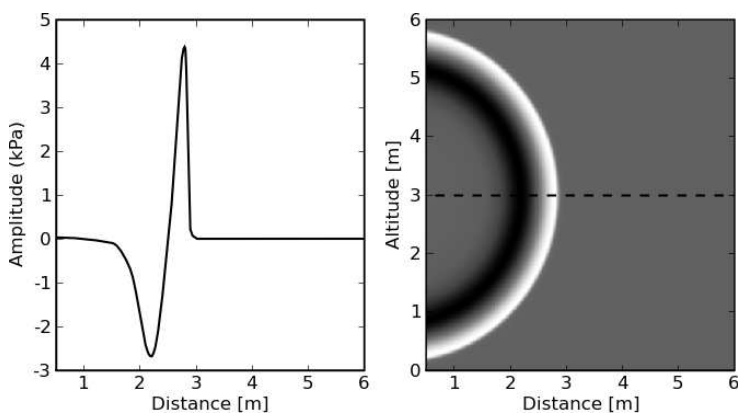

FIG. 3. Left: pressure waveform at altitude $z=3 \mathrm{~m}$. Right: initial pressure waveform used to start the reference and NPE calculations.

of approximately 100 points per wavelength. This signal is then spherically extrapolated to obtain a 2D array. Fig. 3 shows the one dimensional signal and its $2 \mathrm{D}$ extension used to start both reference and NPE calculations.

A simulation on a perfectly rigid ground was performed together with two calculations on different ground layers. Both have identical tortuosity $(\Phi=3)$ and porosity $\left(\Omega_{0}=0.3\right)$ but have different flow resistivity values $\left(\sigma_{0}=100 \mathrm{kPa} . \mathrm{s.m} \mathrm{m}^{-2}\right.$ and $\left.\sigma_{0}=10 \mathrm{kPa} . \mathrm{s} . \mathrm{m}^{-2}\right)$. These flow resistivity values have been chosen to test the model limitations rather than to represent a real situation. Chosen flow resitivities would correspond to grass $\left(\sigma_{0}=100\right.$ $\left.\mathrm{kPa} . \mathrm{s} . \mathrm{m}^{-2}\right)$ and light, dry snow $\left(\sigma_{0}=10 \mathrm{kPa} . \mathrm{s} . \mathrm{m}^{-2}\right)$. The ground layer is $75 \mathrm{~cm}$ thick $(50$ points) and for NPE calculations the moving-window is $4.5 \mathrm{~m}$ wide and $6 \mathrm{~m}$ high (300 by 400 points).

\section{Results}

Fig. 4 shows snapshots of the propagation for non-rigid ground layers at time $t=33 \mathrm{~ms}$ for both models. Colormaps represent results for NPE model while contour lines are results from Euler's equations. Time signals are recorded at the virtual receivers; Fig. 5 shows these signals for NPE and reference calculations for the three ground layers considered. Although the Euler's equations model seems to smear out reflected waves more than the NPE model, the parabolic model produces time waveforms comparable to the references. 

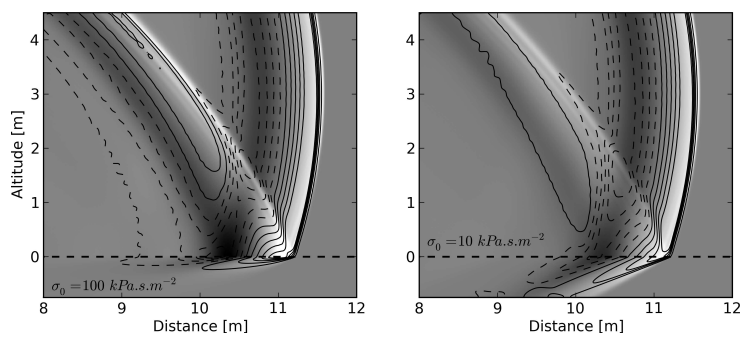

FIG. 4. Snapshots at time $t=33$ ms. Left: $\sigma_{0}=100 \mathrm{kPa} . \mathrm{s} . \mathrm{m}^{-2} ;$ Right: $\sigma_{0}=10 \mathrm{kPa} . \mathrm{s} . \mathrm{m}^{-2}$; Colormap: solution from NPE model; Contour lines: solution from Euler's equations. Ten contour lines equally spaced from -800 to $800 \mathrm{~Pa}$ are shown. Contours corresponding to negative values are represented by dashed lines, positive ones by solid lines.
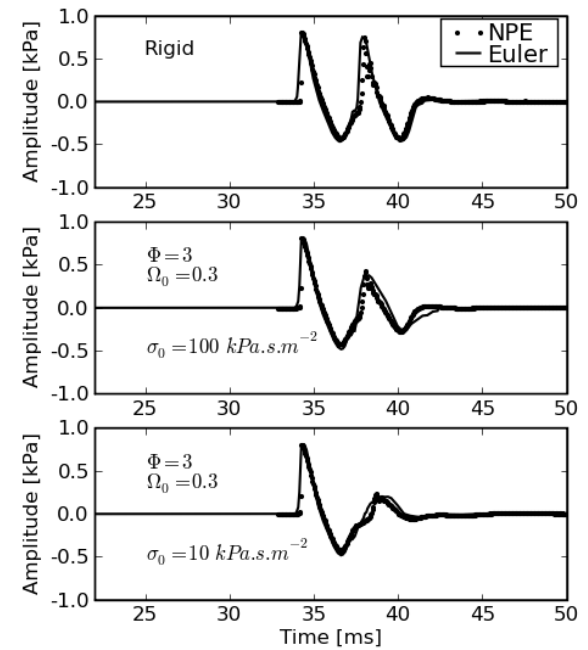

FIG. 5. Time signals at the receiver for NPE and reference calculations for the three ground layers considered (from top to bottom: perfectly rigid, $\sigma_{0}=100 \mathrm{kPa} . \mathrm{s} . \mathrm{m}^{-2}$ and $\sigma_{0}=10$ kPa.s.m ${ }^{-2}$ ). Solid line: Euler; Dotted line: NPE.

To evaluate the accuracy of the NPE model, some characteristics of the reflected wave are studied. Namely, these are the maximum positive and negative peak pressures and their arrival times (noted respectively $p_{+}$and $p_{-}, t_{a+}$ and $t_{a-}$ ), and the positive phase duration $\left(\right.$ noted $t_{d}$ ). These characteristics are sumarized in Table I. Since for the softest ground layer the negative peak on the reflected wave almost does not exist, values of $p_{-}$and $t_{a-}$ for this 
layer are irrelevant.

As one can see, arrival times differ by at most $0.3 \mathrm{~ms}$. The difference is larger for the softest layer; this can be explained by the fact that the NPE model does not smear out pulses as the reference model do, leading to erroneous positive peak position. One can thus expect that as the flow resistivity decreases the error on arrival time increases. However, in outdoor sound propagation applications the flow resitivity may rarely be lower than the one used here $\left(\sigma_{0}=10 \mathrm{kPa} . \mathrm{s} . \mathrm{m}^{-2}\right)$, so the error on arrival time will remain weak for most cases. These remarks are also applicable to the positive phase duration $t_{d}$. Positive peak amplitudes differ by $6.2 \%$ and $5.1 \%$ for layers with $\sigma_{0}=100 \mathrm{kPa} . \mathrm{s} . \mathrm{m}^{-2}$ and $\sigma_{0}=10$ kPa.s.m ${ }^{-2}$, respectively. This difference does not seem to be dependant on flow resitivity and as a comparison, the relative error for the perfectly rigid layer is $1 \%$. Relative error for negative peaks are comparable: these are $2.4 \%$ and $3.4 \%$ for rigid and the layer with $\sigma_{0}=100 \mathrm{kPa} . \mathrm{s} . \mathrm{m}^{-2}$, respectively.

As a mean of comparison, calculation times for Euler and NPE models were about 3.5 hours and 4 minutes, respectively (calculations were done on a modern desktop computer). Although the Euler's equations implementation could use more advanced numerical techniques (adaptive mesh refinement methods ${ }^{25}$, moving window principle ${ }^{15}$ ), the NPE model, thanks to the use of a one-variable one-way wave equation and a fast solver (Thomas algorithm), is a very efficient tool for sound propagation simulations.

\section{INCLUDING FORCHHEIMER'S NONLINEARITIES IN THE TWO-WAY COUPLING}

While the flow resistivity dependance on particle velocity (Forchheimer's nonlinearities) are accounted for in the NPE model for porous ground layers (last term in Eq. (18)), the two-way coupling between both domains does not contain high-amplitude effects on ground properties. This would lead to wrong solutions, since an additional attenuation

would be introduced in the ground layer, but the increased rigidity of the interface wouldn't 
be accounted for.

A solution is to artificially increase the flow resistivity appearing in the coupling parameters Eqs. (29) according to:

$$
\sigma(x, t)=\sigma_{0}\left(1+\xi w^{i}\right)
$$

where $w^{i}$ is the vertical particle velocity at the interface. Note that the flow resistivity is now dependant on $(x, t)$; it is thus noted $\sigma(x, t)$. We then use Eq. (21) to obtain an approximation of $w^{i}$ :

$$
w^{i}=\left(\rho_{0} c_{0}\right)^{-1} \int \partial_{z} p_{1}^{i} d x+O\left(\epsilon^{5 / 2}\right)
$$

where $p_{1}^{i}$ is the first-order approximation of the pressure at the interface. The flow resistivity $\sigma_{0}$ in the coupling parameters Eqs. (29) is thus replaced by:

$$
\sigma(x, t)=\sigma_{0}\left(1+\frac{\xi}{\rho_{0} c_{0}} \int \partial_{z} p_{1}^{i} d x\right)
$$

At the beginning of each time step, the flow resistivity is thus updated with the help of pressure values at the interface at the previous time step. This method, although approximate, allows to include Forchheimer's nonlinearities in the two-way coupling.

\section{A. Numerical example}

To illustrate the effects of Forchheimer's nonlinearities a simulation is performed with a nonlinearity parameter $\xi=2.5 \mathrm{~s} . \mathrm{m}^{-1}$. According to the conclusions of the previous section, low flow resistivities lead to more error on positive phase duration and time of arrival of positive peak pressure. A low flow resitivity has been chosen $\left(\sigma_{0}=10\right.$ kPa.m.s ${ }^{-2}$, with $\left.\phi=3, \Omega_{0}=0.3\right)$, so that the method to include Forchheimer's nonlinearities can be fully evaluated.

Simulation parameters and initilizing array are identical to those used in section IV.B. The source is positioned at $\left(x_{s}, z_{s}\right)=(0,3) m$; the receiver is placed 12 meters away from the source at the same altitude.

Fig. 6 shows time signals at the virtual receiver and Table II sumarizes their charac-

teristics for both reference and NPE calculations. One can see that compared to the same 


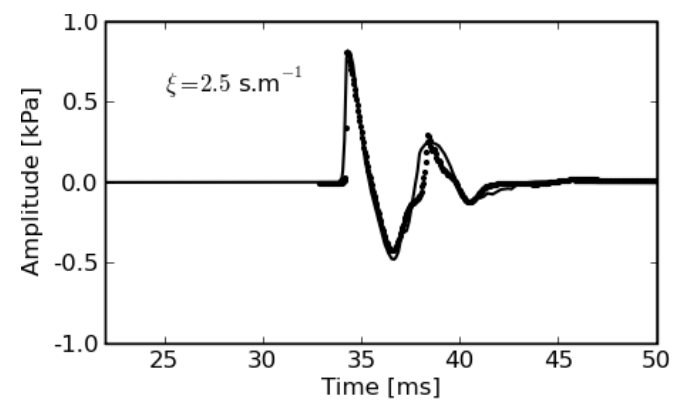

FIG. 6. Time signals at the receiver for a Forchheimer's parameter $\xi=2.5$ s.m ${ }^{-1}$. Solid line: Euler; Dotted line: NPE.

ground layer with no Forchheimer's nonlinearities (bottom subplot in Fig. 5), the obtained reflected wave has a larger positive peak amplitude and a lower time of arrival. The relative error for positive and negative peak pressures are $4.38 \%$ and $4.34 \%$, respectively, while the error on positive phase duration is $0.4 \mathrm{~ms}$. These values, in agreement with the ones found in section IV.B, seem to indicate that the method used to include Forchheimer's nonlinearities in the two way-coupling does not introduce any additional source of error.

To confirm this statement, differences in signals characteristics for calculations with and without nonlinearities are studied. Table III presents these figures for both models. The positive peak amplitude is increased by $21.78 \%$ and $21.12 \%$, and the time of arrival $t_{a+}$ is reduced by 0.7 and $0.6 \mathrm{~ms}$ for Euler and NPE simulations, respectively, while the positive phase duration is reduced by $0.3 \mathrm{~ms}$ for both models. The signals modifications due to the addition of Forchheimer's nonlinearities are nearly identical for both models, confirming that the method presented to take into account the flow resistivity dependance on particle velocity is accurate.

\section{CONCLUSION \& PERSPECTIVES}

A NPE model based on a nonlinear extension of the Zwikker-Kosten model has been derived; it allows to simulate weakly nonlinear propagation within a porous ground layer. 
Next, two-way coupling equations have been derived from linearized Euler's equations. This interfacial boundary condition couples air and ground NPE models and enables the NPE to account for the effects of soft ground layers on sound propagation. For linear propagation, this method has been shown to give very good agreement with analytical solutions for a wide range of ground properties. For high-amplitudes waves, the NPE model produces time signals comparable to those obtained by Euler's equations model. Relative error on peak pressures has been shown to be independant on material properties while differences on positive phase duration and time of arrival increases with decreasing ground flow resitivity. However, the presented model still gives good agreement even for very low flow resitivities and provides a simple but efficient way of taking into account ground impedances. Finally, an approximate method to include Forchheimer's nonlinearities in the two-way coupling is presented: it consists of artificially increasing the flow resistivity value in the coupling parameters. This method has been proven to give satisfactory results and does not introduce any additional source of error in the two-way coupling.

To construct the NPE model, the assumption that the ground layer is equivalent to a continuous fluid has been made. This simplified modeling allows to derive a ground model that is of the same form of the NPE model for atmospheric layer. Two-way coupling equations involving only spatial derivatives and integrals, the complete NPE model is able to perform simulations in very short times (about 50 times faster than the Euler's equations implementation). This enables the model to be later used to study finite-amplitude sound propagation over urban environments, by performing a large number of simulations to get wave field statistics in the air layer. However, note that for realistic simulations, a spherical spreading term should be added to the NPE used in this work.

The relative simplicity of the nonlinear parabolic equation model and its coupling method makes it a good candidate for extensions and modifications. In a previous work ${ }^{35}$, the NPE model for porous ground layers and two-way coupling equations have been adapted to handle non-flat topographies, through the use of terrain-following coordinates ${ }^{19}$. Two-way coupling equations could also be derived for multilayered ground surfaces without much ad- 
ditional work. With atmospheric refraction and dissipation included, it provides a complete NPE model for weakly nonlinear wave propagation including most of the features of sound propagation outdoors (refraction, dissipation, topography and ground impedance effects). This tool can be used for instance for propagating waves from explosions using a three stages procedure: first, a method based on Euler's equations is used in the near field, where the propagation is highly nonlinear. Next, NPE models can propagate weakly nonlinear waves over moderate distances and finally, when the wave amplitude is low enough, frequencydomain method like the PE can be used. This hybrid method allows to propagate waves from explosions over distances up to several kilometers ${ }^{3}$.

\section{Acknowledgments}

The authors would like to thank F. Van Der Eerden and E. Salomons for providing the initialization array and answering our questions about it.

\section{APPENDIX A: NUMERICAL SOLUTION OF EULER'S EQUATIONS}

The WENO scheme has fifth-order accuracy. The main principle of the WENO scheme is the use of multiple stencils to evaluate the derivative at a given point. The algorithm first determines where there is a discontinuity and then weights stencils accordingly to avoid spurious numerical oscillations. This features make the WENO scheme accurate for propagating shock waves. For the sake of brevity computation details are omitted here, but the reader may refer to the work of $\mathrm{Shu}^{33}$ or Wochner ${ }^{16,36}$.

The time discretization scheme is of the form:

$$
\begin{aligned}
\mathbf{w}^{(1)} & =\mathbf{w}^{n}+\Delta t \mathbf{K}^{n}, \\
\mathbf{w}^{(2)} & =\frac{3}{4} \mathbf{w}^{n}+\frac{1}{4} \mathbf{w}^{(1)}+\frac{1}{4} \Delta t \mathbf{K}^{(1)}, \\
\mathbf{w}^{n+1} & =\frac{1}{3} \mathbf{w}^{n}+\frac{2}{3} \mathbf{w}^{(2)}+\frac{2}{3} \Delta t \mathbf{K}^{(2)}
\end{aligned}
$$


where, for the air layer, $\mathbf{w}^{n}$ is the solution vector at time iteration $n$, i.e.:

$$
\mathbf{w}^{n}=\left(\begin{array}{c}
\rho_{T} \\
\rho_{T} u \\
\rho_{T} w \\
\rho_{T} e_{0}
\end{array}\right)^{n}
$$

and $\mathbf{K}^{(i)}$ is the right hand side of the equation system, i.e.:

$$
\begin{aligned}
\mathbf{K}^{(i)}=-\partial_{x}\left(\begin{array}{c}
\rho_{T} u \\
\rho_{T} u^{2} \\
\rho_{T} u w \\
\rho_{T} e_{0} u
\end{array}\right)^{(i)}-\partial_{z}\left(\begin{array}{c}
\rho_{T} w \\
\rho_{T} u w \\
\rho_{T} w^{2} \\
\rho_{T} e_{0} w
\end{array}\right)^{(i)} \\
-\left(\begin{array}{c}
0 \\
\partial_{x} p_{T} \\
\partial_{z} p_{T} \\
-\partial_{x}\left(p_{T} u\right)-\partial_{z}\left(p_{T} w\right)
\end{array}\right)^{(i)}
\end{aligned}
$$

Note that for the ground layer, $\mathbf{w}^{n}$ and $\mathbf{K}^{(i)}$ have to be modified according to Eqs. (35).

Although the combination of WENO and Runge-Kutta schemes allow to stably propagate discontinuities, it is unable to propagate waves of infinite slope: a shock smearing will occur where the slope is too steep, resulting in small deviations from physical solutions for very high amplitude waves.

\section{REFERENCES}

1 B. E. McDonald and W. A. Kuperman, "Time domain formulation for pulse propagation including nonlinear behaviour at a caustic", J. Acoust. Soc. of Am. 81, 1406-1417 (1987).

2 K. Castor, P. Gerstoft, P. Roux, B. McDonald, and W. Kuperman, "Long-range propagation of finite-amplitude acoustic waves in an ocean waveguide", J. Acoust. Soc. of Am. 116, 2004-2010 (2004). 
3 F. van der Eerden and E. Védy, "Propagation of shock waves from source to receiver", Noise Control Eng. J. 53, 87-93 (2005).

4 K. Attenborough, P. Schomer, E. Védy, and F. van der Eerden, "Overview of the theoretical development and experimental validation of blast sound-absorbing surfaces", Noise Control Eng. J. 53, 70-80 (2005).

${ }^{5}$ K. Attenborough, A. Cummings, P. Dutta, P. Schomer, E. Salomons, E. Standley, O. Umnova, F. van den Berg, F. van der Eerden, P. van der Weele, and E. Védy, "Blast sound absorbing surfaces. A joint project of the ERDC and the Netherlands ministry of defense", Technical Report, Engineer Research and Development Center/Cold Regions Research and Engineering Lab (2004).

6 T. Leissing, "Nonlinear outdoor sound propagation - A numerical implementation and study using the nonlinear progressive wave equation", Master's thesis, Chalmers University of Technology, Göteborg (2007).

7 B. E. McDonald, P. Caine, and M. West, "A tutorial on the nonlinear progressive wave equation (NPE) - Part 1", Applied Acoustics 43, 159-167 (1994).

8 B. E. McDonald, "Weak shock interaction with a free-slip interface at low grazing angles", J. Acoust. Soc. of Am. 91, 718-733 (1992).

9 P. Caine and M. West, "A tutorial on the nonlinear progressive wave equation (NPE) Part 2. derivation of the three dimensional cartesian version without use of perturbation expansions", Applied Acoustics 45, 155-165 (1995).

10 G. P. Too and J. H. Ginsberg, "Cylindrical and spherical coordinate versions of the NPE for transient and steady-state sound beams", J. Vib. Acoust. 114, 420-424 (1992).

11 G. P. J. Too and S. T. Lee, "Thermoviscous effects on transient and steady-state sound beams using nonlinear progressive wave equation models", J. Acoust. Soc. of Am. 97, 867-874 (1995).

12 B. E. McDonald, "High-angle formulation for the nonlinear progressive wave equation model", Wave Motion 31, 165-171 (2000).

13 J. J. Ambrosiano, D. R. Plante, B. E. McDonald, and W. A. Kuperman, "Nonlinear 
propagation in an ocean acoustic waveguide", J. Acoust. Soc. of Am. 87, 1473-1481 (1990).

14 A. A. Piacsek, "Atmospheric turbulence conditions leading to focused and folded sonic boom wave fronts", J. Acoust. Soc. of Am. 111, 520-529 (2002).

15 V. W. Sparrow and R. Raspet, "A numerical method for general finite amplitude wave propagation in two dimensions and its application to spark pulses", J. Acoust. Soc. of Am. 90, 2683-2691 (1991).

16 M. S. Wochner, A. A. Atchley, and V. W. Sparrow, "Numerical simulation of finite amplitude wave propagation in air using a realistic atmospheric absorption model", J. Acoust. Soc. of Am. 118, 2891-2898 (2005).

17 D. Heimann and R. Blumrich, "A linearized Eulerian sound propagation model for studies of complex meteorological effects", J. Acoust. Soc. of Am. 112, 446-455 (2002).

18 V. E. Ostashev, D. K. Wilson, L. Liu, D. F. Aldridge, N. P. Symons, and D. Marlin, "Equations for finite-difference, time-domain simulation of sound propagation in moving inhomogeneous media and numerical implementation", J. Acoust. Soc. of Am. 117, 503$517(2005)$.

19 R. Karle and D. Heimann, "A linearized eulerian finite-difference time-domain sound propagation model with terrain-following coordinates", J. Acoust. Soc. of Am. 119, 38133821 (2006).

20 E. M. Salomons, R. Blumrich, and D. Heimann, "Eulerian time-domain model for sound propagation over a finite impedance ground surface. Comparison with frequency-domain models", Acta Acustica United With Acustica 88, 483-492 (2002).

21 D. K. Wilson, V. E. Ostashev, S. L. Collier, N. P. Symons, D. F. Aldridge, and D. H. Marlin, "Time-domain calculations of sound interactions with outdoor ground surfaces", Appl. Acoust. 68, 173-200 (2007).

22 D. K. Wilson, S. L. Collier, V. E. Ostashev, D. F. Aldridge, N. P. Symons, and D. H. Marlin, "Time-domain modeling of the acoustic impedance of porous surfaces", Acustica United With Acta Acustica 95, 965-975 (2006). 
23 D. K. Wilson, V. E. Ostashev, and S. L. Collier, "Time-domain equations for sound propagation in rigid-frame porous media", J. Acoust. Soc. of Am. 116, 1889-1892 (2004).

24 J. Berenger, "A perfectly matched layer for the absorption of electromagnetic waves", J. Comput. Phys. 114, 185-200 (1994).

25 T. Plewa, Adaptive Mesh Refinement - Theory and Applications: Proceedings of the Chicago Workshop on Adaptive Mesh Refinement Methods, Sept. 3-5, 2003 (Springer, Berlin) (2005).

26 P. H. Forchheimer, "Wasserbewegung durch boden", Z. Ver. Dtsch. Ing. 45, 1782-1788 (1901).

27 D. K. Wilson, J. D. McIntosh, and R. F. Lambert, "Forchheimer-type nonlinearities for high-intensity wave propagation of pure tones in air-saturated porous media", J. Acoust. Soc. of Am. 84, 350-359 (1988).

28 C. Zwikker and C. W. Kosten, Sound absorbing materials (Elsevier, New York) (1949).

29 A. Krylov, S. Sorek, A. Levy, and G. Ben-Dor, "Simple waves in saturated porous media (I. The isothermal case)", The Japan Soc. of Mech. Eng. Int. Journal 39, 294-298 (1996).

30 E. Védy, "Simulations of flows in porous media with a flux corrected transport algorithm", Noise Control Eng. J. 50, 211-217 (2002).

31 O. Umnova, K. Attenborough, and A. Cummings, "High amplitude pulse propagation and reflection from a rigid porous layer", Noise Control Eng. J. 50, 204-210 (2002).

32 X. Di and L. E. Gilbert, "An exact laplace transform for a point sorce above a ground surface", J. Acoust. Soc. of Am. 93, 714-720 (1993).

33 Essentially non-oscillatory schemes for hyperbolic conservation laws, volume 1697, chapter in B. Cockburn, C. Johnson, C.-W. Shu, and E. Tadmor (Edited by A. Quarteroni), Advanced Numerical Approximation of Nonlinear Hyperbolic Equations, Lecture Notes in Mathematics, 325-432 (Springer, Berlin) (1998).

34 W. Press, B. Flannery, S. Teukolsky, and W. Vetterling, eds., Numerical recipes in Fortran, chapter 16, 702-708 (Cambridge University Press, Cambridge) (1996).

35 T. Leissing, P. Jean, J. Defrance, and C. Soize, "Nonlinear parabolic equation model for 
finite-amplitude sound propagation in an inhomogeneous medium over a nonflat, finiteimpedance ground surface", in Proceedings of EuroNoise 08, 1889-1894 (Paris) (2008). 36 M. S. Wochner, "Numerical simulation of multi-dimensional acoustic propagation in air including the effects of molecular relaxation", Ph.D. thesis, The Pennsylvania State University (2006). 
TABLE I. Reflected wave characteristics for reference and NPE calculations.

\begin{tabular}{|c|c|c|c|c|c|}
\hline Model & $\begin{array}{c}t_{a+} \\
{[\mathrm{ms}]}\end{array}$ & $\begin{array}{c}p_{+} \\
{[\mathrm{Pa}]}\end{array}$ & $\begin{array}{c}t_{d} \\
{[\mathrm{~ms}]}\end{array}$ & $\begin{array}{c}t_{a-} \\
{[\mathrm{ms}]}\end{array}$ & $\begin{array}{c}p_{-} \\
{[\mathrm{Pa}]}\end{array}$ \\
\hline \multicolumn{6}{|c|}{ Rigid layer: } \\
\hline Euler & 37.9 & 756 & 1.4 & 40.2 & -449 \\
\hline NPE & 38.0 & 749 & 1.3 & 40.0 & -438 \\
\hline \multicolumn{6}{|c|}{$\sigma_{0}=100 \mathrm{kPa} . \mathrm{s} . \mathrm{m}^{-2}:$} \\
\hline Euler & 38.1 & 387 & 1.8 & 40.3 & -265 \\
\hline NPE & 38.2 & 411 & 1.6 & 40.2 & -274 \\
\hline \multicolumn{6}{|c|}{$\sigma_{0}=10 \mathrm{kPa} . \mathrm{s} . \mathrm{m}^{-2}:$} \\
\hline Euler & 39.3 & 202 & 2.4 & - & - \\
\hline NPE & 39 & 213 & 2.0 & - & - \\
\hline
\end{tabular}


TABLE II. Reflected wave characteristics for reference and NPE calculations with Forchheimer's nonlinearities.

\begin{tabular}{lccccc}
\hline \hline Model & $\begin{array}{c}t_{a+} \\
{[\mathrm{ms}]}\end{array}$ & $\begin{array}{c}p_{+} \\
{[\mathrm{Pa}]}\end{array}$ & $\begin{array}{c}t_{d} \\
{[\mathrm{~ms}]}\end{array}$ & $\begin{array}{c}t_{a-} \\
{[\mathrm{ms}]}\end{array}$ & $\begin{array}{c}p_{-} \\
{[\mathrm{Pa}]}\end{array}$ \\
\hline & & & & \\
Euler & 38.6 & 246 & 2.1 & 40.4 & 115 \\
NPE & 38.4 & 258 & 1.7 & 40.5 & 120 \\
\hline \hline
\end{tabular}


TABLE III. Differences in reflected waves characteristics with and without Forchheimer's nonlinearities. Results are shown for both NPE and reference calculations.

\begin{tabular}{lccc}
\hline \hline Model & $t_{a+}$ & $p_{+}$ & $t_{d}$ \\
& {$[\mathrm{~ms}]$} & {$[\mathrm{Pa}]$} & {$[\mathrm{ms}]$} \\
\hline & & & \\
Euler & -0.7 & $+21.78 \%$ & -0.3 \\
NPE & -0.6 & $+21.12 \%$ & -0.3 \\
\hline \hline
\end{tabular}




\section{LIST OF FIGURES}

FIG. 1 The air-ground interface is taken to be midway between two vertical grid points with indexes $j=0$ and $j=1$. Auxiliary virtual points (black circles) with pressure values $p_{i, 0}^{\prime a}$ and $p_{i, 1}^{\prime g}$ are created. . . . . . . . . 10

FIG. 2 SPLs relative to free field at the receiver for the three different ground layers, for NPE and analytical solutions. The source and receiver are placed at altitude $z_{s}=1.4 \mathrm{~m}$; the receiver is $10 \mathrm{~m}$ away from the source. . . . . .

FIG. 3 Left: pressure waveform at altitude $z=3 \mathrm{~m}$. Right: initial pressure waveform used to start the reference and NPE calculations. . . . . . . . . . . . 17

FIG. 4 Snapshots at time $t=33 \mathrm{~ms}$. Left: $\sigma_{0}=100 \mathrm{kPa} . \mathrm{s} . \mathrm{m}^{-2} ;$ Right: $\sigma_{0}=10$ kPa.s.m ${ }^{-2}$; Colormap: solution from NPE model; Contour lines: solution from Euler's equations. Ten contour lines equally spaced from -800 to 800 $\mathrm{Pa}$ are shown. Contours corresponding to negative values are represented by dashed lines, positive ones by solid lines. . . . . . . . . . . . . . . 18

FIG. 5 Time signals at the receiver for NPE and reference calculations for the three ground layers considered (from top to bottom: perfectly rigid, $\sigma_{0}=100$ kPa.s.m ${ }^{-2}$ and $\sigma_{0}=10$ kPa.s.m ${ }^{-2}$ ). Solid line: Euler; Dotted line: NPE. . . 18

FIG. 6 Time signals at the receiver for a Forchheimer's parameter $\xi=2.5$ s.m ${ }^{-1}$. Solid line: Euler; Dotted line: NPE. . . . . . . . . . . . . . . . . . . . . 21 